\title{
Assessment of perioperative critical cerebral and splanchnic desaturation periods during neonatal heart surgery
}

\author{
Van Ooteghem M. ${ }^{1}$, Moerman A. ${ }^{1}$, De Hert S. ${ }^{1}$, François K. ${ }^{2}$, Vandekerckhove K. ${ }^{3}$, Peperstraete H. ${ }^{4}$ \\ Ghent University Hospital, Dept of Anaesthesiology ${ }^{1}$, Cardiac surgery², Paediatrics ${ }^{3}$ and Intensive Care ${ }^{4}$, Gent, \\ Belgium
}

\section{Aim of the study:}

To identify critical cerebral and splanchnic desaturation periods during neonatal heart surgery.

\section{Material and Methods:}

- Prospective observational study in neonatal heart surgery with and without the use of cardiopulmonary bypass.

- Cerebral and splanchnic saturation was measured with near-infrared spectroscopy (Nonin Equanox ${ }^{\mathrm{TM}}$ 7600).

- Eleven potential jeopardizing events were evaluated.

- Differences between the two surgical groups were evaluated with the independent samples t-test. Changes over time were assessed with a linear mixed model.

\section{Results and Discussion:}

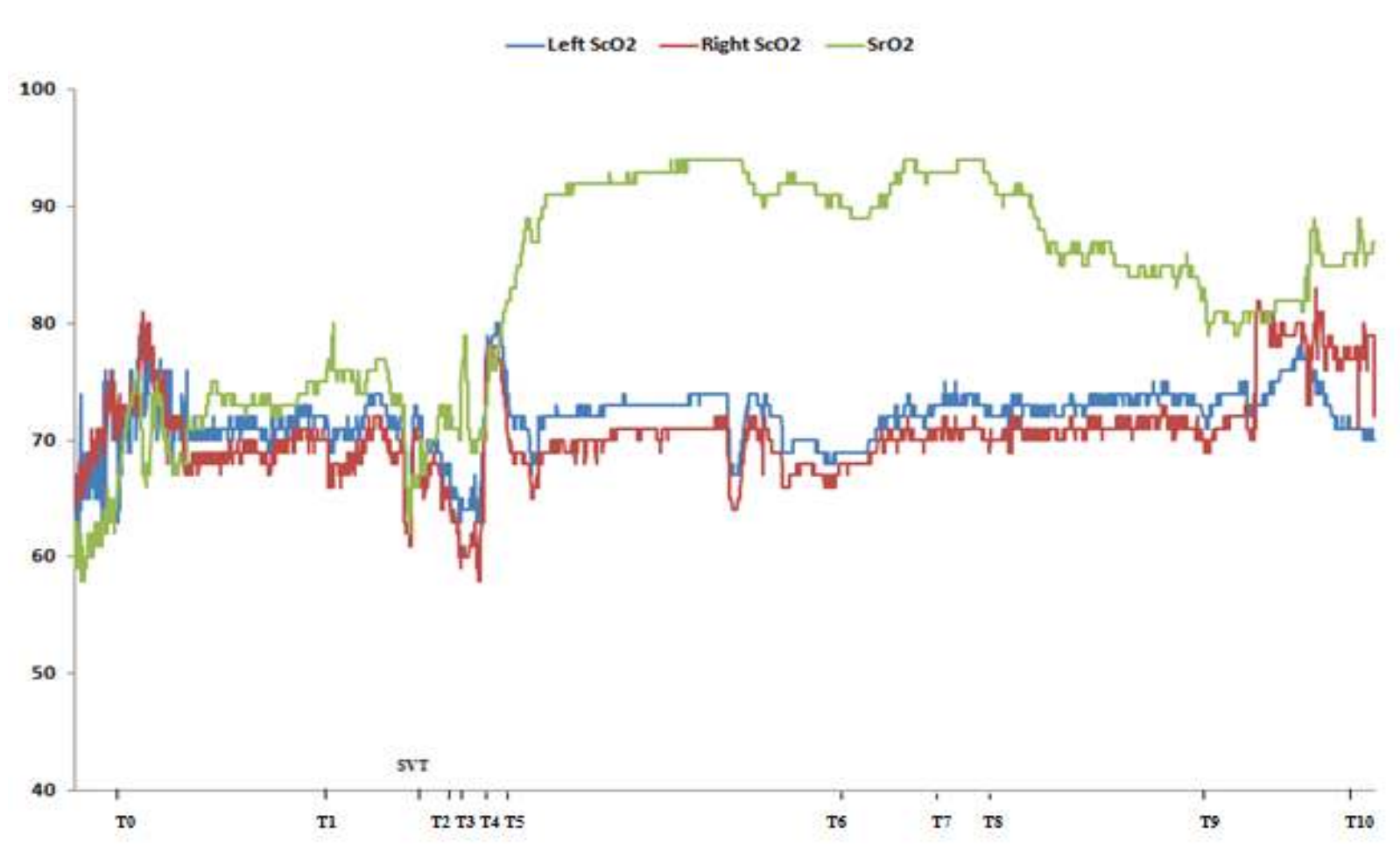

Figure 1: Typical intraoperative course of NIRS values during arterial switch repair

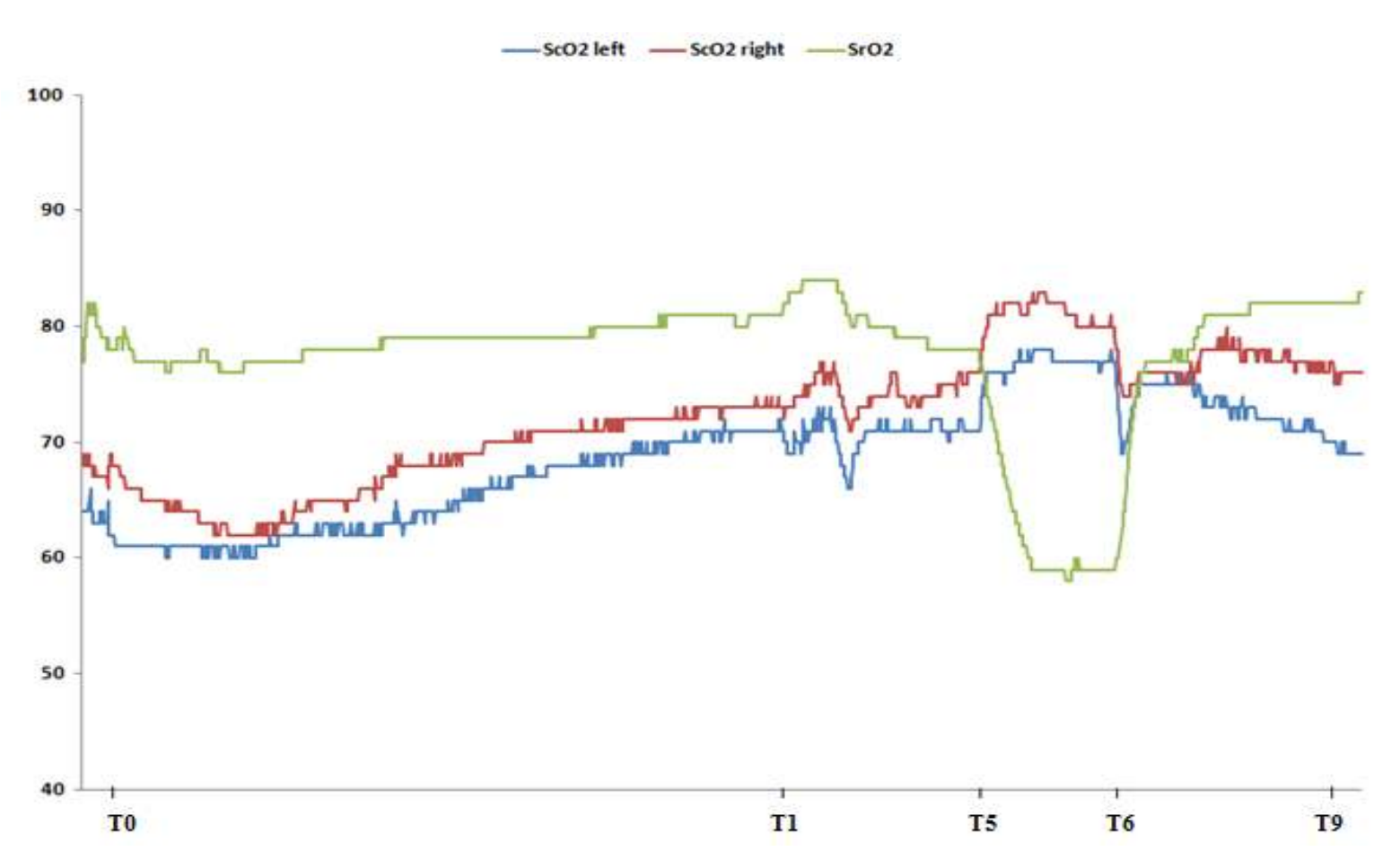

Figure 2: Typical intraoperative course of NIRS values during aortic coarctation repair
- 12 neonates undergoing arterial switch repair and 11 coarctation patients were studied.

- Two patients $(9 \%)$ had an absolute decrease in cerebral saturation below $50 \%$. Eight patients (35\%) showed a relative decrease of $>20 \%$ from baseline.

- The two groups were only significantly different during aortic cross-clamping (Cerebral saturation: $72 \pm 8 \%$ vs $80 \pm 5 \%, p=0.012$, and renal saturation: $84 \pm 12 \%$ vs $54 \pm 11 \%, \quad p<0.001$, for arterial switch repair and coarctation repair, respectively) (Figure 3 ).

- Main risk moments for cerebral desaturation in the arterial switch group were arterial and venous cannulation and start of cardiopulmonary bypass.

- Risk moments in both groups were transfer to intensive care and extubation.
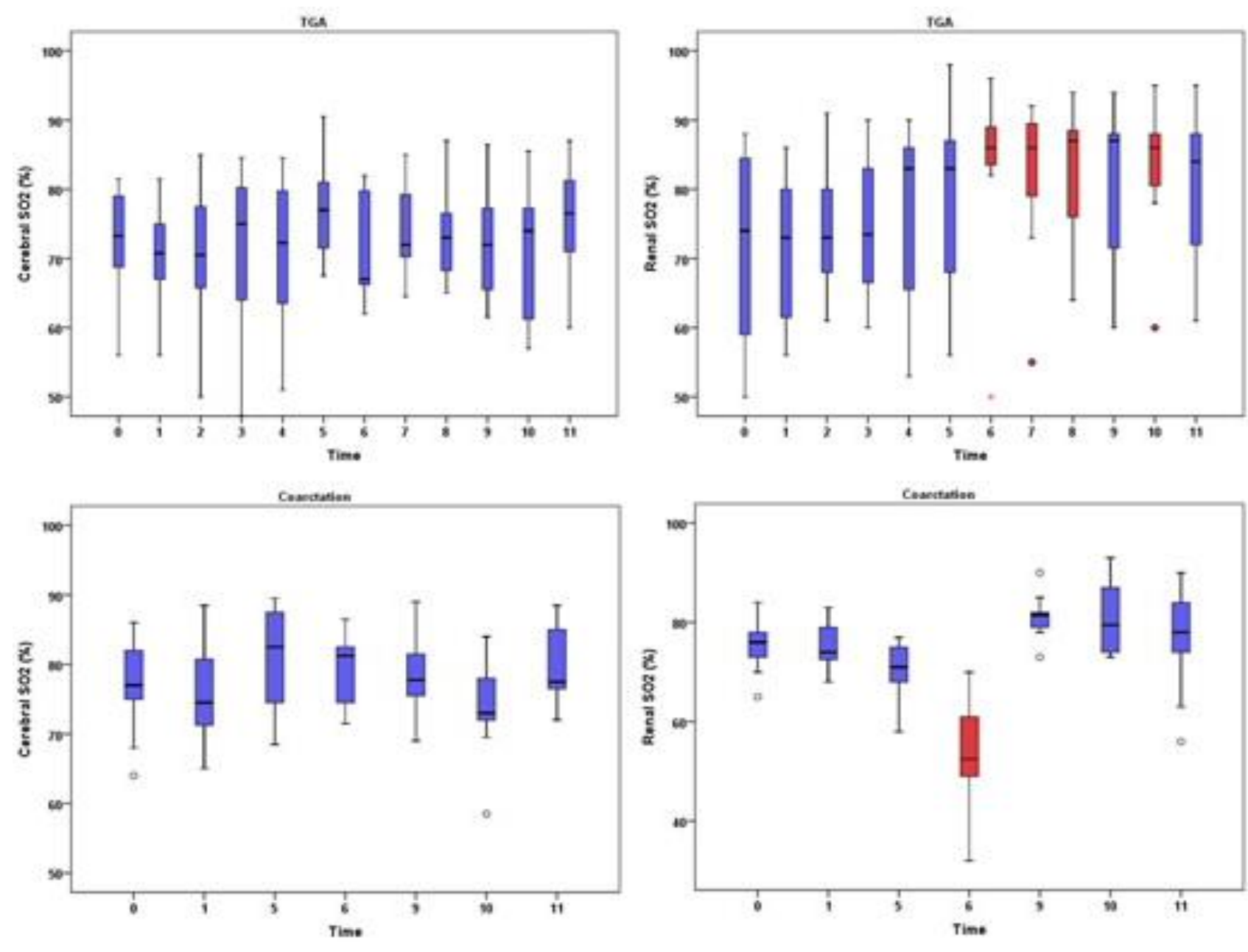

Figure 3: Boxplot of NIRS values during predefined critical events (Red boxes: significantly different from baseline)

\section{Conclusion:}

Cerebral and splanchnic desaturations were short lasting and linked to specific, predictable perioperative events. 